\title{
NGHIÊN CỨU SỰ ĐÁNH ĐỒI GIŨA LợI ÍCH VÀ CHI PHÍ KHI SỦ DỤNG DİCH VỤ TRỰC TUYẾN: TRƯỜNG HỢP THƯƠNG MẠI DI ĐỘNG TẠI VIẸT NAM
}

\author{
BÙI THÀNH KHOA ${ }^{1}$, NGUYẼ̃N MINH HÀ ${ }^{2}$ \\ ${ }^{1}$ Trường Đại học Công nghiệp Thành phố Hồ Chí Minh; \\ ${ }^{2}$ Trường Đại học Mở Thành phố Hồ Chí Minh; \\ buithanhkhoa@iuh.edu.vn,ha.nm@ou.edu.vn
}

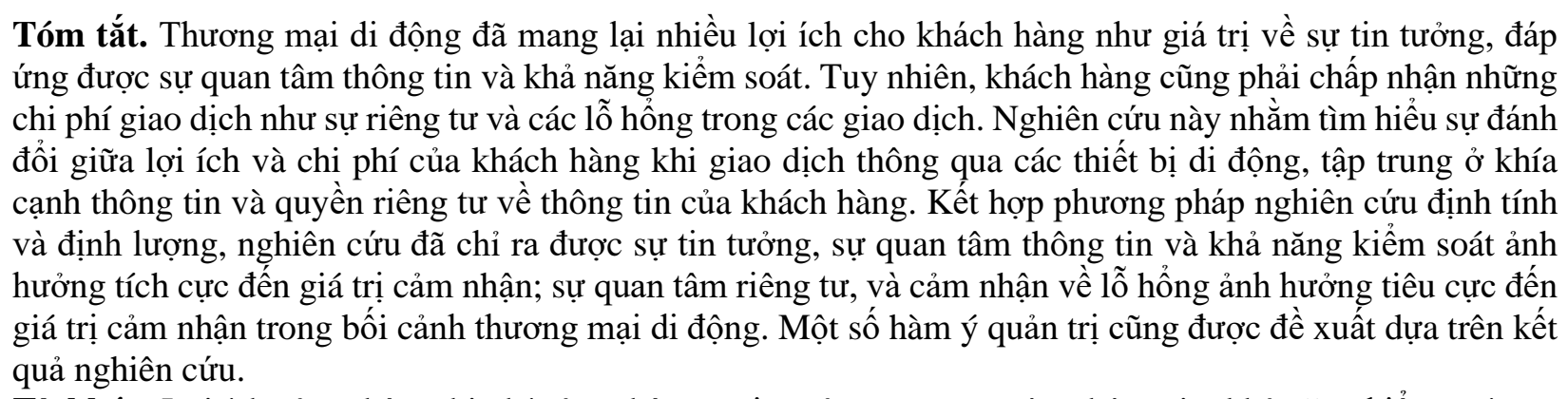

Từ khóa. Lợi ích cảm nhận, chi phí cảm nhận, sự tin tưởng, sự quan tâm thông tin, khả năng kiểm soát, sự quan tâm riêng tư, nhận thức về lỗ hổng, thương mại di động.

\section{A STUDY ON TRADE-OFF BETWEEN BENEFIT AND COST WHEN USING ONLINE SERVICES: CASE OF MOBILE COMMERCE IN VIETNAM}

\begin{abstract}
Mobile commerce (m-commerce) has brought many benefits to customers such as trust, the information interest, and ability control. However, customer must also accept transaction costs such as privacy concern and perceived vulnerability in the mobile commerce transaction. This study aims to explore the trade-off between customers' benefit and cost when trading through mobile devices, focusing on the information and privacy of customer. With qualitative research and quantitative research method, the study has shown trust, information interest, and control ability to affect perceived values positively; privacy concern and perceived vulnerability negatively affect perceived value in the context of mobile commerce. Some managerial implications are also proposed based on the research result.
\end{abstract}

Keywords. Perceived benefit, perceived cost, trust, information interest, control ability, privacy concern, perceived vulnerability, m-commerce

\section{GIÓ́I THIỆU}

Thương mại di động là một phần mở rộng của thương mại điện tử, với việc giao dịch thông qua việc sử dụng nền tảng ứng dụng di động và mạng không dây [1]. Tính linh động của thương mại di động cho phép các hoạt động giao dịch diễn ra bất cứ lúc nào và bất cứ nơi nào [2]. Sự phát triển nhanh chóng của công nghệ không dây bao gồm cả thiết bị và đường truyền đang thúc đẩy thương mại di động trở thành một loại hình giao dịch phổ biến giữa doanh nghiệp và người tiêu dùng [3].

Theo báo cáo khảo sát của Nielsen, tỷ lệ người dùng điện thoại thông minh tại các thành phố trọng tâm chiếm $84 \%$ vào năm 2017, tăng 6\% so với năm 2016 [4]. Khi nói đến các thiết bị được người Việt sử dụng để kết nối Internet, $31 \%$ thường xuyên truy cập bằng điện thoại thông minh và $38 \%$ sử dụng máy tính xách tay của họ [5]. Có đến 75\% người dưới 35 tuổi sử dụng điện thoại thông minh thay vì máy tính cho mục đích giải trí, tìm kiếm thông tin, kết nối mạng xã hội, mua sắm. Có thể nói, sự gia tăng tỷ lệ người dùng điện thoại thông minh tại Việt Nam cùng với sự phát triển của Internet, mạng di động $3 \mathrm{G}, 4 \mathrm{G}$ là cơ 
hội để thương mại di động bùng nổ [6] . Hơn 49\% doanh nghiệp nhận đơn đặt hàng qua ứng dụng trên di động và con số này được dự đoán sẽ còn tăng rất nhanh trong thời gian tới. Riêng với những trang thương mại điện tử lớn như Lazada thì doanh thu từ việc bán hàng qua di động cũng chiếm từ 50-60\% doanh thu tổng. Do đó, dịch chuyển sang nền tảng di động là xu hướng tất yếu của thương mại điện tử [7].

Turner và Gellman [8] cho rằng lợi ích cảm nhận liên quan đến nhận thức về các kết quả tích cực là do một hành động cụ thể. Theo Forsythe và cộng sụ [9], trong lĩnh vực thương mại điện tử, lợi ích cảm nhận chỉ ra những gì khách hàng thu được từ mua sắm trực tuyến. Nói cách khác, Ko và cộng sư [10] cho rằng lợi ích cảm nhận là niềm tin của người tiêu dùng về việc họ có thể mua sắm bất kỳ thời gian nào mà không có bất kỳ khó khăn hoặc thậm chí bị gián đoạn trong quá trình mua sắm. Tổng lợi ích của khách hàng là giá trị được nhận thức bao gồm các thành phần lợi ích kinh tế, chức năng và tâm lý mà khách hàng mong đợi từ một người bán nhất định dựa trên sản phẩm, dịch vụ, con người và hình ảnh được cung cấp [11]. Chi phí cảm nhận bao gồm tiền thanh toán và các khoản không phải là tiền như thời gian, những nỗ lực phải bỏ ra [12]. Theo khái niệm của Zeithaml [13], chi phí được khách hàng được đánh giá thông qua cảm nhận những gì mà họ đã, đang và sẽ mất mát khi giao dịch. Theo Ahola và cộng sụ [14], tổng chi phí khách hàng bao gồm chi phí tiền tệ, chi phí thời gian, chi phí năng lượng và chi phí tinh thần.

Thương mại di động đã mang lại nhiều lợi ích cho người tiêu dùng. Các lợi ích này bao gồm tính linh động (có thể giao dịch bất cứ ở đâu và bất cứ khi nào), tính thuận tiện và khả năng hoạt động (các chức năng của một chiếc điện thoại thông minh ngày càng tỷ lệ nghịch với kích thước và giá tiền của chúng), tính tương tác (hệ thống di động cho phép khách hàng tương tác nhanh và dễ dàng hơn với các thiết bị khác), tính cá nhân hóa (điện thoại thông minh là một thiết bị cá nhân, có ghi thông tin và thói quen mua sắm của từng người), và tính vị trí hóa (thông qua mạng lưới định vị toàn cầu, nhà cung cấp dịch vụ thương mại di động biết được vị trí của người dùng trong thời gian thực cung cấp cơ hội cung cấp cho họ quảng cáo di động, phiếu giảm giá hoặc các dịch vụ khác có liên quan) [15]. Các yếu tố tiện lợi của thương mại di động góp phần tạo ra các lợi ích như xây dựng sự tin tưởng của khách hàng, đáp ứng đúng quan tâm về thông tin, và tăng khả năng kiểm soát thông tin của khách hàng. Tuy nhiên, thương mại di động vẫn còn một số các hạn chế ảnh hưởng đến chi phí khách hàng phải chấp nhận, đặc biệt ở khía cạnh thông tin. Đặc tính cá nhân hóa của thương mại di động được đánh giá là lợi ích tuy nhiên cũng là một yếu tố chi phí đối với người dùng. Hơn nữa, chi phí cảm nhận trong thương mại di động có thể là nhận thức về sự mất mát về chất lượng sản phẩm, bảo mật dữ liệu hoặc đánh cắp thông tin khi tham gia một giao dịch [16]. Ngoài ra, các rủi ro bảo mật và quyền riêng tư khi sử dụng ứng dụng di động với phương tiện không dây cũng được xem là đặc thù chi phí của thương mại di động khi các giao dịch và thông tin được diễn ra trong thời gian thực (real-time) [17]. Ngày nay, các thiết bị cầm tay có sức mạnh tương đương với các máy tính để bàn trước đó. Hơn nữa, các thiết bị cầm tay cũng được hỗ trợ các chức năng tương đồng với máy tính bàn dẫn đến rủi ro bảo mật cho máy tính để bàn cũng tương đồng cho các thiết bị không dây. Ví dụ, mã độc, một vấn đề ảnh hưởng đến máy tính để bàn cũng trở thành một một vấn đề nghiêm trọng đối với các thiết bị không dây cầm tay [17].

Nghiên cứu sự đánh đổi giữa lợi ích và chi phí khi sử dụng các dịch vụ trực tuyến, ứng dụng trường hợp thương mại di động liên quan đến vấn đề thông tin của khách hàng, nhằm khám phá mối quan hệ giữa các yếu tố về lợi ích cảm nhận (sự tin tưởng, sự quan tâm thông tin, và khả năng kiểm soát) với các yếu tố về chi phi cảm nhận (quan tâm về sự riêng tư, nhận thức về lỗ hổng) trong việc tạo ra giá trị cảm nhận cho khách hàng khi mua hàng qua các thiết bị và ứng dụng di động tại Việt Nam. Từ đó, nghiên cứu sẽ đề xuất một số hàm ý quản trị cho các doanh nghiệp kinh doanh trong lĩnh vực thương mại di động.

\section{CO' SỞ LÝ THUYẾT}

Sự đánh đổi được định nghĩa là mất hoặc giảm một thứ gì đó để đổi lấy việc tăng hoặc thu được những thứ khác để có thể cải thiện tối đa hóa tổng lợi nhuận hoặc hiệu quả trong các trường hợp cụ thể 
[18]. Phù hợp với học thuyết kỳ vọng, khách hàng khi mua hàng từ các ứng dụng di động sẽ nỗ lực để tối đa hóa kết quả tích cực và giảm thiểu kết quả tiêu cực [19]. Do đó, khách hàng sẽ đưa ra quyết định đánh đổi khi đánh giá nguy cơ vi phạm quyền riêng tư và rủi ro thông tin có xứng đáng với lợi ích của trải nghiệm cá nhân hóa, tăng thêm thông tin hoặc tăng khả năng kiểm soát. Mối quan tâm riêng tư, sự đáng tin của doanh nghiệp, cảm nhận liên quan về thông tin cung cấp cũng như lợi ích sẽ ảnh hưởng đến giá trị cảm nhận khi mua hàng trực tuyến [20]. Ở lĩnh vực hệ thống thông tin, các nhà nghiên cứu đã chỉ ra mối quan hệ giữa lợi ích cảm nhận và chi phí cảm nhận có sự liên quan đến hành vi sử dụng hoặc chấp nhận thương mại điện tử [21,22]; các nghiên cứu liên quan đến giá trị cảm nhận, đặc biệt, lĩnh vực thương mại di động còn hạn chế. Nghiên cứu này đã dựa trên các nghiên cứu trước đây, cũng như tích hợp các quan điểm và kiểm tra thực nghiệm các yếu tố xây dựng giá trị cảm nhận của khách hàng ở bối cảnh thương mại điện tử làm cơ sở để khái niệm về giá trị cảm nhận của người tiêu dùng trong bối cảnh thương mại di động tại Việt Nam. Mô hình lý thuyết được trình bày như Hình 1.

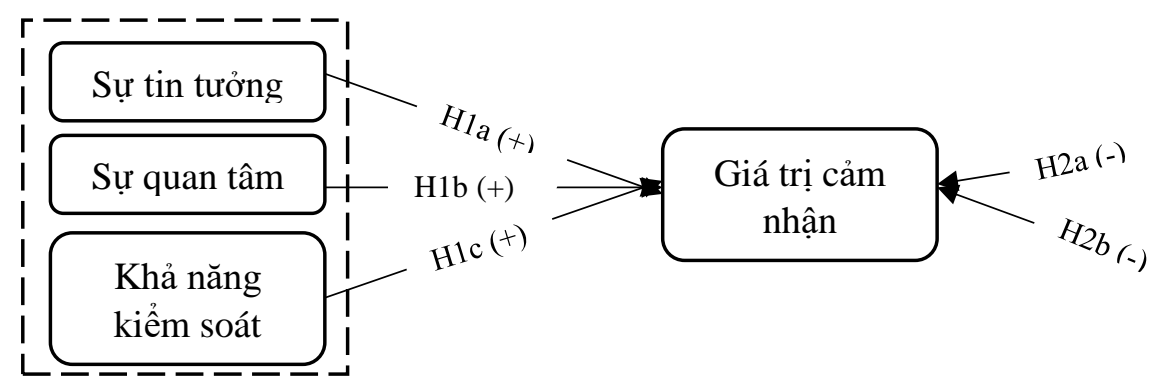

Lọi ích cảm nhận

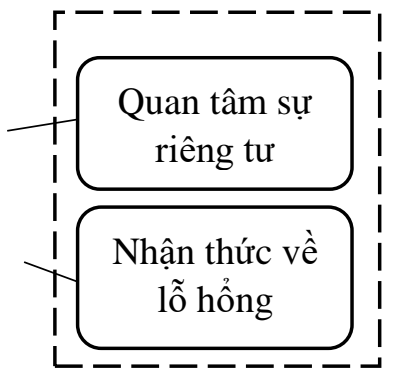

Chi phí cảm nhận

Hình 1. Mô hình lý thuyết

Giá trị cảm nhận là khái niệm gồm hai phần, một phần là các lợi ích thu được (kinh tế, xã hội và quan hệ) và một phần là những chi phí bỏ ra (giá, thời gian, nỗ lực, rủi ro và sự tiện lợi) của khách hàng [23]. Giá trị cảm nhận là sự đánh giá tổng thể của người tiêu dùng về tiện ích của một sản phẩm hay dịch vụ dựa vào nhận thức của họ về những gì nhận được và những gì phải bỏ ra [13]. Một số người tiêu dùng cảm nhận được giá trị khi có một mức giá thấp, những người khác cảm nhận được giá trị khi có một sự cân bằng giữa chất lượng và giá cả [24]. Như vậy, những người tiêu dùng khác nhau, các thành phần của giá trị cảm nhận có thể là khác biệt. Do đó, đây là một khái niệm mang tính cá nhân và chủ quan [25]. Trong nghiên cứu này, mô hình nghiên cứu được xem như một mô hình nguyên nhân (formative measure model), trong đó, cấu trúc giá trị cảm nhận là một biến tiềm ẩn được tạo ra từ các biến quan sát về lợi ích cảm nhận và chi phí cảm nhận khi cung cấp thông tin trong giao dịch thương mại di động [26, 27].

Sự tin tưởng là một trong những yếu tố giúp mọi người hợp tác với nhau. Sự tin tưởng là khách hàng tin rằng người bán sẽ hành động như họ mong đợi [28]. Một yếu tố quan trọng để tăng sự tin tưởng của người tiêu dùng là cung cấp các sản phẩm và dịch vụ tốt, phù hợp nhu cầu và đạt được sự ủng hộ nhiều hơn của khách hàng [21]. Tuy nhiên, quá trình xây dựng niềm tin cũng phụ thuộc vào thông tin khách hàng cung cấp, điều này có thể cản trở sự phát triển niềm tin. Việc cung cấp các dịch vụ có chất lượng, phù hợp với từng khách hàng sẽ gia tăng niềm tin cho khách hàng; tuy nhiên, đó cũng là quá trình tăng cường thu thập thông tin từ khách hàng [29]. Niềm tin được sử dụng như một yếu tố lợi ích mà khách hàng sẽ cảm thấy thoải mái và an toàn khi sử dụng dịch vụ thương mại di động của khách hàng. Bên cạnh đó, danh tiếng của nhà bán hàng cũng là một trong các yếu tố tạo ra lợi ích khi góp phần xây dựng sự tự tin trong cung cấp thông tin của khách hàng khi giao dịch trực tuyến qua thiết bị di động [30].

Một yếu tố lợi ích khác ảnh hưởng đến việc mua sắm trực tuyến đó chính là sự quan tâm thông tin của khách hàng [31]. Sự quan tâm thông tin xảy ra khi người dùng tập trung vào hệ thống trực tuyến hoặc 
một ứng dụng di động của trang thương mại điện tử với cảm xúc thú vị [32]. Nghiên cứu trước đây đã phát hiện ra rằng sự quan tâm là một yếu tố cơ bản dẫn đến hành vi sử dụng công nghệ trực tuyến của khách hàng như sử dụng công nghệ thư điện tử, truy cập các trang thương mại điện tử, ý định quay lại trang thương mại điện tử và phát triển trở thành khách hàng thân thiết $[33,34]$. Sự quan tâm là một yếu tố tích cực trong thái độ của người dùng đối với một hệ thống, giúp nhận ra thái độ của khách hàng khi họ mua sắm trực tuyến [35]. Sự quan tâm thông tin khi mua sắm qua các thiết bị di động của khách hàng sẽ tạo ra sự tìm hiểu về cách thức sử dụng ứng dụng, các quy định trong việc giao dịch, cũng như cung cấp thông tin cho người làm marketing để nhận được thông tin về sản phẩm/dịch vụ phù hợp với nhu cầu của bản thân [21]. Sự quan tâm thông tin được xem như một lợi ích cho khách hàng nhằm để doanh nghiệp tạo ra những chiến dịch marketing cá nhân hóa phù hợp với từng khách hàng.

Theo Goffman [36], cá nhân có thể quyết định cách họ có thể gây ấn tượng với người khác khi sở hữu khả năng kiểm soát thông tin cá nhân. Khả năng kiểm soát có thể được thể hiện bằng sức mạnh để hạn chế một số thông tin cá nhân [37]và quyết định sử dụng thông tin [38]. Khách hàng không thể chấp nhận được nếu các nhà tiếp thị sử dụng thông tin cá nhân của họ để kiếm thêm tiền [39]. Khách hàng nên được thông báo về việc sử dụng thêm thông tin của họ [40]. Khi sử dụng các thiết bị di động để mua hàng, khách hàng có thể kiểm soát được thông tin họ nhập vào và trả về từ hệ thống. Đặc biệt, với các thiết bị có độ bảo mật cao, lợi ích này càng được gia tăng. Hơn nữa, khả năng kiểm soát cũng là một vấn đề quan trọng góp phần quyết định mua hàng của khách hàng [41]. Vì vậy, gia tăng khả năng kiểm soát thông tin cho khách hàng đồng nghĩa với việc gia tăng về lợi ích cảm nhận cho họ và tạo ra sự yên tâm trong mua sắm thông qua các thiết bị di động.

Quan tâm về riêng tư trên Internet cung cấp cho khách hàng tự tin khi tham gia vào các giao dịch trực tuyến. Với mức độ gia tăng kinh nghiệm sử dụng Internet, người dùng có nhiều khả năng nhận thức được các biện pháp bảo vệ thông tin riêng tư và có xu hướng quan hệ tích cực giao dịch trực tuyến [42]. Các nhà nghiên cứu ngành hệ thống thông tin đã nghiên cứu về mối quan tâm riêng tư trong khía cạnh thông tin, định nghĩa về quyền riêng tư là quyền tự mình kiểm soát thông tin cá nhân $[21,43]$. Mối quan tâm về quyền riêng tư đề cập đến quan điểm của một khách hàng về giá trị của tình huống tiết lộ thông tin cá nhân, người dùng sẽ xem xét sự cân bằng khi trao đổi thông tin cá nhân của họ [44]. Mối quan tâm thông tin riêng tư và liên kết nhận thức cá nhân với chế độ đảm bảo bảo mật cho thấy rằng mối quan tâm riêng tư của khách hàng về việc có thể mất sự riêng tư như là kết quả của việc nhận thức về vấn đề phải tiết lộ thông tin cho một đại lý bên ngoài [45]. Trong giao dịch thương mại di động, các khách hàng phải cung cấp các thông tin như số điện thoại, địa chỉ email, địa chỉ nhà, thậm chí cho phép các ứng dụng di động đọc thông tin của tài khoản mạng xã hội, danh bạ điện thoại, hình ảnh. Sự quan tâm riêng tư đã được chứng minh nghịch biến với cung cấp thông tin, việc chấp nhận giảm mối quan tâm về sự riêng tư trong giao dịch trực tuyến thể hiện sự đánh đổi để đạt được kết quả mong muốn của khách hàng [46].

Khách hàng mua hàng trên các ứng dụng di động cũng đồng nghĩa với việc chấp nhận một số rủi ro thông tin có thể xảy ra. Việc nhận thức các rủi ro này được gọi là nhận thức về lỗ hổng trong giao dịch [47]. Mối quan tâm về thông tin của một khách hàng liên quan đến năm vấn đề bao gồm việc thu thập mà không được sự đồng ý của người dùng, sử dụng nội bộ thông tin khách hàng mà không được phép, sử dụng thông tin khách hàng cho bên ngoài mà không được phép, thông tin khách hàng bị sử dụng bởi một người không được phép trong tổ chức và bị lỗi dẫn đến lộ thông tin khách hàng [48]. Trừ vấn đề đầu tiên thuộc về mối quan tâm riêng tư, bốn khía cạnh sau của an ninh thông tin thuộc về phía trách nhiệm của người bán. Những hậu quả tiêu cực mà người tiêu dùng có thể gặp khi bị xâm phạm quyền riêng tư bao gồm việc đánh cắp thông tin nhận dạng, hồ sơ cá nhân và quảng cáo dựa trên việc sử dụng thứ cấp của người tiêu dùng (remarketing hoặc retargeting) [49].Theo Culnan và Milberg [50], khách hàng sẽ cảm thấy lo lắng về việc mất thông tin cá nhân khi họ sử dụng công nghệ trực tuyến, bởi vì họ khó có thể giám sát, lưu giữ, tìm kiếm thông tin cá nhân. Tuy nhiên, đây là các rủi ro nằm ngoài sự kiểm soát của người mua, và thông thường 
khách hàng sẽ phụ thuộc vào các cam kết về an ninh cũng như chính sách riêng tư của người bán hàng. Các rủi ro khi giao dịch trong thương mại điện tử nói chung và thương mại di động nói riêng được xem như chi phí hoặc đầu ra đối với người tiêu dùng [17].

Để đánh giá mức độ đánh đổi của người dùng về thông tin, Hann và cộng sụ [51] đã xây dựng một các kích thích kết hợp hai ưu đãi kinh tế (phần thưởng tiền tệ và sự thuận tiện trong tương lai) và ba mối lo ngại về quyền riêng tư (lỗi lưu trữ hoặc xử lý thông tin cá nhân, sử dụng thông tin thứ cấp trái phép và truy cập thông tin không đúng cách). Kết quả chỉ ra rằng mối quan tâm của cá nhân đối với quyền riêng tư không phải là tuyệt đối, mà là họ sẵn sàng đánh đổi những lo ngại về quyền riêng tư vì lợi ích kinh tế. Giả thuyết cơ bản của lý chuỗi phương tiện - giá trị là hành vi của người tiêu dùng thường được hướng theo mục tiêu và có thể được coi là động lực của người tiêu dùng thông qua hệ thống phân cấp mục tiêu [52]. Trong một hệ thống phân cấp như vậy, các giá trị có thể được coi là mục tiêu cuối cùng thúc đẩy người tiêu dùng tham gia vào hành vi mua sắm, và lợi ích là các mục tiêu phụ phụ thuộc vào các giá trị [53]. Khách hàng đạt được các lợi ích tích lũy từ các thuộc tính [54]. Xu và cộng sụ [45] thấy rằng, mặc dù các cơ hội lớn được cung cấp bởi nhận biết vị trí trong marketing (LAM), mối quan tâm riêng tư của khách hàng sử dụng thiết bị di động xuất hiện như một yếu tố ngăn cản trong việc họ chấp nhận LAM. Nghiên cứu mở rộng mô hình tính toán riêng tư để khám phá những nghịch lý cá nhân, đặc biệt trong LAM, với những cân nhắc các đặc điểm cá nhân và hai cách tiếp cận với từng cá nhân (bí mật và công khai). Chi phí kinh tế của một quyết định không tốt là yếu tố rủi ro thường được thảo luận, cũng như nghiên cứu nhiều nhất. Những khách hàng mua qua điện thoại biết rằng cô ấy có thể trả lại một món hàng nếu họ chứng minh được sản phẩm đó không đạt yêu cầu; do đó, có thể khách hàng không nhận thấy bất kỳ rủi ro nào về thiệt hại tài chính. Tuy nhiên, khách hàng ấy có thể cảm thấy nguy cơ đáng kể về mất thời gian (phải trả lại việc mua hàng, trì hoãn việc có được một món đồ cần thiết); mất thể diện và thất vọng (sự không hài lòng do mua hàng kém); và không đạt được mục tiêu mua của mình $[55,56]$. Thông qua nghiên cứu thực nghiệm cho thấy những ảnh hưởng của chi phí cảm nhận và lợi ích cảm nhận sẽ khác nhau đối với mỗi cá nhân và kỳ vọng của họ. Từ đó, ta có giả thuyết:

H1a: Sự tin tưởng có ảnh hưởng tích cực đến giá trị cảm nhận khi cung cấp thông tin trong mua hàng trong thương mại di động

H1b: Sự quan tâm thông tin có ảnh hưởng tích cực đến giá trị cảm nhận khi cung cấp thông tin trong mua hàng trong thương mại di động

H1c: Khả năng kiểm soát có ảnh hưởng tích cực đến giá trị cảm nhận khi cung cấp thông tin trong mua hàng trong thương mại di động

H2a: Quan tâm sự riêng tư có ảnh hưởng tiêu cực đến giá trị cảm nhận khi cung cấp thông tin trong mua hàng trong thương mại di động

H2b: Nhận thức về lỗ hổng có ảnh hưởng tiêu cực đến giá trị cảm nhận khi cung cấp thông tin trong mua hàng trong thương mại di động

\section{PHƯƠNG PHÁP NGHIÊN CÚU}

Phương pháp nghiên cứu hỗn hợp bao gồm phương pháp nghiên cứu định tính và phương pháp nghiên cứu định lượng được sử dụng để đạt được mục tiêu nghiên cứu đề ra. Nghiên cứu định tính được thực hiện nhằm xác định các yếu tố được xem như là chi phí và lợi ích thông tin khi mua hàng qua các thiết bị di động cũng như dùng để điều chỉnh lại bảng câu hỏi. Phương pháp thu thập dữ liệu trong nghiên cứu định tính là thảo luận nhóm tập trung $[57,58]$. Đối tượng tham gia thảo luận bao gồm 10 khách hàng được chọn bằng phương pháp phát triển mầm, có kinh nghiệm mua sắm trong thương mại di động trên một năm. Cuộc thảo luận được diễn ra dưới sự chủ trì của tác giả với một dàn bài hướng dẫn thảo luận. Kết quả nghiên cứu định tính cho thấy chi phí mà 10 khách hàng nhận thấy bao gồm sự đánh đổi thông tin riêng tư và lo lắng về những lỗ hổng về thông tin; và, những lợi ích cảm nhận được bao gồm sự tin tưởng, đạt được thông 
tin họ quan tâm và khả năng kiểm soát thông tin khi giao dịch thông qua các thiết bị di động. Bên cạnh đó, tất cả người tham gia thảo luận đều nhất trí rằng chi phí cảm nhận có tác động tiêu cực đến giá trị cảm nhận, còn lợi ích cảm nhận sẽ làm gia tăng giá trị cảm nhận của họ với một ứng dụng mua sắm trực tuyến. Ngoài ra, các thang đo trong nghiên cứu về sự tin tưởng, sự quan tâm thông tin, khả năng kiểm soát, quan tâm sự riêng tư, cũng như nhận thức về lỗ hổng trong lĩnh vực thương mại di động cũng được điều chỉnh cho phù hợp với suy nghĩ của các đối tượng khảo sát nói riêng và người mua hàng Việt Nam nói chung. Bước tiếp theo, bảng câu hỏi được thiết kế để tiến hành nghiên cứu định lượng. Để đạt được mục tiêu nghiên cứu của đề tài, kỹ thuật chọn mẫu trong nghiên cứu này chọn mẫu phi xác suất, kỹ thuật lấy mẫu theo mục đích. Vì nghiên cứu đi chuyên sâu về lĩnh vực thương mại di động, nên áp dụng lấy mẫu theo mục đích sẽ chọn được đối tượng khảo sát hiểu rõ về vấn đề nghiên cứu và từ đó có thể mở rộng mẫu dễ dàng [59].

Bảng1. Thông tin mẫu nghiên cứu

\begin{tabular}{|c|l|c|c|}
\hline $\begin{array}{c}\text { Tiêu } \\
\text { thức }\end{array}$ & \multicolumn{1}{|c|}{ Phân loại } & $\begin{array}{c}\text { Số } \\
\text { lượng }\end{array}$ & $\begin{array}{c}\text { Phần } \\
\text { trăm }\end{array}$ \\
\hline \multirow{2}{*}{$\begin{array}{c}\text { Giới } \\
\text { tính }\end{array}$} & Nam & 296 & 49,6 \\
\cline { 2 - 4 } & Nữ & 301 & 50,4 \\
\hline \multirow{4}{*}{$\begin{array}{c}\text { Độ } \\
\text { tuồi }\end{array}$} & Dưới 20 tuổi & 71 & 11,9 \\
\cline { 2 - 4 } & $20-24$ & 115 & 19,3 \\
\cline { 2 - 4 } & $25-29$ & 165 & 27,6 \\
\cline { 2 - 4 } & $30-34$ & 138 & 23,1 \\
\hline
\end{tabular}

\begin{tabular}{|c|c|c|c|}
\hline Tiêu thức & Phân loại & $\begin{array}{c}\text { Số } \\
\text { lượng }\end{array}$ & $\begin{array}{l}\text { Phần } \\
\text { trăm }\end{array}$ \\
\hline \multirow{7}{*}{$\begin{array}{l}\text { Nghề } \\
\text { nghiệp }\end{array}$} & Học sinh - sinh viên & 71 & 11,9 \\
\hline & Giảng viên & 90 & 15,1 \\
\hline & Nhân viên văn phòng & 171 & 28,6 \\
\hline & Nội trợ & 69 & 11,6 \\
\hline & Buôn bán & 89 & 14,9 \\
\hline & Công chức & 88 & 14,7 \\
\hline & Công nhân & 19 & 3,2 \\
\hline \multirow{4}{*}{ Học vấn } & Trung học & 127 & 21,3 \\
\hline & Trung cấp - Cao đẳng & 208 & 34,8 \\
\hline & Đại học & 113 & 18,9 \\
\hline & Sau Đại học & 149 & 25,0 \\
\hline
\end{tabular}

Bảng 2. Thống kê các nhân tố sử dụng trong nghiên cứu

\begin{tabular}{|l|c|l|l|}
\hline \multicolumn{1}{|c|}{ Nhóm nhân tố } & $\begin{array}{c}\text { Số lượng } \\
\text { biến }\end{array}$ & \multicolumn{1}{|c|}{ Nguồn } & \multicolumn{1}{|c|}{$\begin{array}{c}\text { Thang đo } 5 \text { bậc (điểm giữa là } \\
\text { trung tính) }\end{array}$} \\
\hline Sự tin tưởng (TR) & 3 & $\begin{array}{l}\text { Dinev và Hart [30], Kowatsch và } \\
\text { Maass [61]; thảo luận nhóm. }\end{array}$ & $\begin{array}{l}\text { (1) Hoàn toàn không đồng ý } \\
\text { (5) Hoàn toàn đồng ý }\end{array}$ \\
\hline Khả năng kiểm soát (AC) & 4 & $\begin{array}{l}\text { Martin và công } s u ̛ \text { [62]; } \\
\text { thảo luận nhóm. }\end{array}$ & $\begin{array}{l}\text { (1) Hoàn toàn không đồng ý } \\
\text { (5) Hoàn toàn đồng ý }\end{array}$ \\
\hline Sự quan tâm thông tin (IN) & 3 & $\begin{array}{l}\text { Dinev và Hart [30]; } \\
\text { thảo luận nhóm }\end{array}$ & $\begin{array}{l}\text { (1) Hoàn toàn không đồng ý } \\
\text { (5) Hoàn toàn đồng ý }\end{array}$ \\
\hline Quan tâm sự riêng tư (PC) & 4 & $\begin{array}{l}\text { Xu và cọng sụ [63]; } \\
\text { thảo luận nhóm. }\end{array}$ & $\begin{array}{l}\text { (1) Hoàn toàn không quan tâm } \\
\text { (5) Hoàn toàn quan tâm }\end{array}$ \\
\hline $\begin{array}{l}\text { Nhận thức về lỗ hổng } \\
\text { (PAV) }\end{array}$ & 4 & $\begin{array}{l}\text { Smith và cộng sự [48]; } \\
\text { thảo luận nhóm. }\end{array}$ & $\begin{array}{l}\text { (1) Rủi ro rất thấp } \\
\text { (5) Rủi ro rất cao }\end{array}$ \\
\hline
\end{tabular}

Trong 630 phiếu khảo sát được phát ra, có 615 phiếu được thu về. Sau khi sàn lọc và loại bỏ những phiếu khảo sát không hợp lệ (thiếu thông tin, lựa chọn đáp án vi phạm), số phiếu còn lại để đưa vào phân tích định lượng là 597 phiếu, phù hợp và đáng tin cậy để phân tích [60]. Về cụ thể đối tượng trả lời khảo sát, không có sự chênh lệch nhiều về tỷ lệ nam $(49,6 \%)$ và nữ $(50,4 \%)$. Đối tượng tham gia trả lời bảng câu 
hỏi tập trung là nhân viên văn phòng $(28,6 \%)$, giảng viên $(15,1 \%)$, công chức $(14,7 \%)$, người mua bán kinh doanh $(14,9 \%)$ và học sinh - sinh viên $(11,9 \%)$, còn lại là nội trợ, công nhân. Độ tuổi đối tượng khảo sát nằm trong khoảng $20-45$ chiếm $88,1 \%$ và học vấn từ trung cấp trở lên là 470 người chiếm $78,7 \%$. Như vậy, mẫu nghiên cứu phù hợp cho mục đích nghiên cứu; từ đó sẽ góp phần giúp cho nghiên cứu có đánh giá phù hợp về mô hình nghiên cứu.Thang đo của tất cả các khái niệm nghiên cứu trong bài dựa trên các nghiên cứu trước đây, được điều chỉnh qua nghiên cứu sơ bộ và thể hiện dưới dạng các phát biểu.

\section{KẾT QUẢ NGHIÊN CÚU}

Bảng 3. Kết quả thống kê mô tả

\begin{tabular}{|c|c|c|c|c|}
\hline Thang đo & Ký hiệu & $\begin{array}{l}\text { Giá trị trung } \\
\text { bình }\end{array}$ & Skewness & Kurtosis \\
\hline $\begin{array}{l}\text { Các ứng dụng di động có danh tiếng là môi trường an toàn để trao đổi } \\
\text { thông tin với người khác. }\end{array}$ & TR1 & 3,79 & $-0,824$ & $-0,151$ \\
\hline $\begin{array}{l}\text { Các ứng dụng di động là môi trường đáng tin cậy để thực hiện các giao } \\
\text { dịch kinh doanh. }\end{array}$ & TR2 & 3,78 & $-0,845$ & $-0,211$ \\
\hline $\begin{array}{l}\text { Các ứng dụng di động xử lý thông tin cá nhân được gửi bởi người dùng } \\
\text { một cách đúng thẩm quyền }\end{array}$ & TR3 & 3,72 & $-0,916$ & 0,147 \\
\hline Tôi tin tôi kiểm soát những gì xảy ra với thông tin cá nhân của tôi & $\mathrm{AC} 1$ & 3,41 & $-0,545$ & $-0,383$ \\
\hline Mức độ thông tin được công ty sử dụng tùy theo ý muốn của tôi & $\mathrm{AC} 2$ & 3,66 & $-0,754$ & $-0,181$ \\
\hline Tôi có thể biết thông tin của tôi sẽ được sử dụng như thế nào & $\mathrm{AC} 3$ & 3,60 & $-0,811$ & $-0,021$ \\
\hline Tôi có thể biết thông tin của tôi được chia sẻ cho ai & $\mathrm{AC} 4$ & 3,62 & $-0,812$ & 0,065 \\
\hline $\begin{array}{l}\text { Sự quan tâm đối với thông tin mà tôi muốn có cao hơn mối quan tâm } \\
\text { về sự riêng tư hay lỗ hổng thông tin }\end{array}$ & IN1 & 3,49 & $-0,451$ & $-0,549$ \\
\hline $\begin{array}{l}\text { Sự quan tâm của tôi để có được một thông tin nhất định làm tôi quên đi } \\
\text { sự quan tâm riêng tư của mình }\end{array}$ & IN2 & 3,44 & $-0,416$ & $-0,635$ \\
\hline $\begin{array}{l}\text { Nói chung, nhu cầu của tôi để có được thông tin cụ thể từ Internet lớn } \\
\text { hơn mối quan tâm của tôi về quyền riêng tư }\end{array}$ & IN3 & 3,34 & $-0,313$ & $-0,637$ \\
\hline Tôi cảm thấy phiền khi ứng dụng hỏi nhiều về thông tin cá nhân & PC1 & 2,50 & 0,532 & $-0,808$ \\
\hline $\begin{array}{l}\text { Tôi quan tâm rằng các ứng dụng mua hàng đang thu thập quá nhiều } \\
\text { thông tin cá nhân của tôi }\end{array}$ & PC2 & 2,54 & 0,477 & $-0,783$ \\
\hline $\begin{array}{l}\text { Tôi quan tâm đến việc thông tin của tôi có được sử dụng đúng với mục } \\
\text { đích ban đầu mà ứng dụng mua hàng nêu ra }\end{array}$ & PC3 & 2,59 & 0,405 & $-0,882$ \\
\hline $\begin{array}{l}\text { Tôi quan tâm về việc liên kết thông tin về tài khoản mạng xã hội cho } \\
\text { ứng dụng mua hàng }\end{array}$ & PC4 & 2,56 & 0,382 & $-0,762$ \\
\hline $\begin{array}{l}\text { Thông tin của tôi cung cấp có thể được sử dụng cho mục đích quảng } \\
\text { cáo của công ty }\end{array}$ & PAV1 & 2,61 & 0,437 & $-0,911$ \\
\hline Thông tin của tôi cung cấp có thể bị bán cho một đơn vị thứ ba & PAV2 & 2,65 & 0,319 & $-0,979$ \\
\hline $\begin{array}{l}\text { Thông tin của tôi cung cấp có thể bị xâm phạm bởi nhân viên không có } \\
\text { thẩm quyền }\end{array}$ & PAV3 & 2,62 & 0,423 & $-0,904$ \\
\hline Thông tin của tôi có thể bị đánh cắp từ hệ thống của công ty & PAV4 & 2,67 & 0,322 & $-0,893$ \\
\hline
\end{tabular}

Theo kết quả thống kê mô tả ở bảng 3 , các nhân tố thuộc lợi ích cảm nhận như sự tin tưởng, khả năng kiểm soát, sự quan tâm thông tin đều trên giá trị trung bình (thấp nhất là 3,34). Trong khi đó các nhân tố thuộc chi phí cảm nhận như quan tâm sự riêng tư và nhận thức về lỗ hổng đều có giá trị trung bình thấp 
(cao nhất là 2,503). Điều này chứng tỏ khách hàng khi mua hàng trong thương mại di động nhận thấy lợi ích của việc cung cấp thông tin và chấp nhận đánh đổi sự riêng tư cũng như các rủi ro có thể xảy ra khi cung cấp thông tin trên ứng dụng di động. Ngoài ra, giá trị Skewness và Kurtosis của tất cả các biến đạt yêu cầu phân phối chuẩn với skewness nhỏ hơn 3, kurtosis nhỏ hơn 10 [64]. Như vậy, tất cả các biến đạt yêu cầu về phân phối chuẩn để thực hiện các phân tích tiếp theo.

Bảng 4. Kết quả kiểm định thang đo cho từng thang đo trong mô hình

\begin{tabular}{|c|c|c|c|c|c|}
\hline Thang đo & CA & FL & $\lambda$ & $\mathbf{C R}$ & AVE \\
\hline \multicolumn{6}{|c|}{ Lọ̣i ích cảm nhận } \\
\hline \multicolumn{6}{|c|}{ KMO $=0,878$; Sig. $($ Bartlett's Test of Sphericit $)=0,00 ;$ Phương sai trích $=67,731 ;$ Eigenvalues $=1,259$} \\
\hline Sự tin cậy & 0,899 & & & $\mathbf{0 , 9}$ & $\mathbf{0 , 7 0 5}$ \\
\hline TR1 & & 0,844 & 0,846 & & \\
\hline TR2 & & 0,836 & 0,877 & & \\
\hline TR3 & & 0,905 & 0,874 & & \\
\hline Khả năng kiểm soát & $\mathbf{0 , 9 3 8}$ & & & 0,938 & 0,791 \\
\hline $\mathrm{AC} 1$ & & 0,864 & 0,861 & & \\
\hline $\mathrm{AC} 2$ & & 0,867 & 0,869 & & \\
\hline $\mathrm{AC} 3$ & & 0,926 & 0,935 & & \\
\hline $\mathrm{AC} 4$ & & 0,885 & 0,89 & & \\
\hline Sự quan tâm thông tin & 0,702 & & & 0,703 & 0,441 \\
\hline IN1 & & 0,784 & 0,675 & & \\
\hline IN2 & & 0,558 & 0,626 & & \\
\hline IN3 & & 0,615 & 0,69 & & \\
\hline
\end{tabular}

Chi phí cảm nhận

KMO = 0,910; Sig. (Bartlett's Test of Sphericit)=0,00; Phương sai trích=63,716; Eigenvalues $=1,071$

\begin{tabular}{ccccc}
\hline Quan tâm sự riêng tự & $\mathbf{0 , 8 6 3}$ & & $\mathbf{0 , 8 6 3}$ & $\mathbf{0 , 6 1 3}$ \\
PC1 & & 0,799 & 0,807 & \\
PC2 & 0,818 & 0,803 & \\
PC3 & 0,734 & 0,765 & \\
PC4 & 0,700 & 0,756 & \\
Cảm nhận về lỗ hổng & $\mathbf{0 , 8 8 6}$ & & & $\mathbf{0 , 8 8 9}$ \\
PAV1 & & 0,785 & 0,807 & \\
PAV2 & 0,805 & 0,803 & \\
PAV3 & 0,822 & 0,765 & \\
PAV4 & 0,765 & 0,756 & \\
\hline
\end{tabular}

CA: Cronbach’s Alpha; FL: Hệ số tải nhân tố; AVE: Tổng phương sai trích; CR: Độ tin cậy tổng hợp; $\lambda$ : trọng số hồi quy chuẩn hóa CFA

Trong nghiên cứu này sẽ kiểm định thang đo của từng thành phần nghiên cứu bao gồm lợi ích cảm nhận (sự tin cậy, khả năng kiểm soát, và sự quan tâm thông tin), chi phí cảm nhận (quan tâm sự riêng tư, và nhận thức về lỗ hổng), và toàn bộ mô hình. Theo bảng 4 , tất cả các thang đo đều đạt độ tin cậy, hệ số tin cậy nằm trong khoảng 0,702 đến 0,938 , đều lớn hơn 0,70 [65]. Kết quả phân tích nhân tố (EFA - principal components with varimax rotation) cho thấy các thang đo đạt yêu cầu về trọng số $\mathrm{KMO}(>0,5)$, kiểm định Bartlett (sig. $>0,05)$, phương sai trích $(>50 \%)$, Eigenvalues $(>1,0)$ và số lượng các nhân tố trích (hệ số tải nhân tố $>0,5)$. Vì vậy các thang đo này có thể sử dụng cho nghiên cứu. Bên cạnh đó, tất cả trọng số hồi quy chuẩn hóa $(\lambda)$ đều lớn hơn 0,5 (thấp nhất là 0,626$)$ và đạt ý nghĩa thống kê $(\mathrm{p}<0,001)$, nên các thang đo trong mô hình đều đạt được giá trị hội tụ [66]. Mức độ tin cậy của thang đo cunng được kiểm tra qua việc tính toán hệ số tin cậy tổng hợp (CR) và tổng phương sai trích (AVE) [67]. Tất cả thang đo đều đạt yêu cầu 
(CR > 0,6, AVE > 0,5), ngoại trừ thang đo sự quan tâm thông tin có AVE là 0,441 , tuy nhiên nếu tổng phương sai trích nhỏ hơn 0,5 , nhưng độ tin cậy tổng hợp cao hơn 0,6 thì hiệu lực hội tụ của cấu trúc vẫn đủ [67]. Như vậy, các thang đo đều đạt mức độ tin cậy, độ nhất quán trong từng thang đo và giá trị hội tụ.

Bảng 5 đã chỉ ra được mức độ phù hợp của các cấu trúc lợi ích cảm nhận, chi phí cảm nhận và toàn bộ mô hình phù hợp với thông tin thị trường [68]. Trong đó, không giống như CFI, TLI không được định mức trong phạm vi 0 đến 1 , do đó, TLI được phép về mặt toán học là > 1 [69]. Ngoài ra, các sai số của các biến quan sát trong các mô hình không có tương quan nhau, nên các thang đo đạt yêu cầu về tính đơn hướng [70].

Bảng 5. Mức độ phù hợp với thông tin thị trường

\begin{tabular}{llllcccc}
\hline Mô hình & \multicolumn{1}{c}{$\chi^{2}$} & \multicolumn{1}{c}{$\mathrm{df}$} & $\chi^{2} / \mathrm{df}$ & GFI & TLI & CFI & RMSEA \\
\hline Lợi ích cảm nhận & 52,773 & 32 & 1,649 & 0,983 & 0,992 & 0,995 & 0,033 \\
Chi phí cảm nhận & 18,048 & 19 & 0,950 & 0,993 & 1,001 & 1,000 & 0,000 \\
Mô hình lý thuyết & 150,081 & 125 & 1,201 & 0,973 & 0,996 & 0,996 & 0,018 \\
\hline
\end{tabular}

Tiếp theo, hệ số tương quan giữa các thành phần của mô hình nghiên cứu với sai lệch chuẩn kèm theo $(\mathrm{r}(\mathrm{se}))$ thấp nhất bằng $0,496(0,036)$ nhỏ hơn $1(\mathrm{p}<0,001$, bảng 6). Vì vậy các khái niệm trong mô hình nghiên cứu đạt giá trị phân biệt [70].

Bảng 6. Hệ số tương quan giữa các khái niệm

\begin{tabular}{|c|c|c|c|c|c|c|}
\hline \multicolumn{3}{|c|}{ Mối quan hệ } & Ước lượng (r) & Sai số chuẩn (SE) & $(1-\mathrm{r}) / \mathrm{SE}$ & Mức ý nghĩa (p) \\
\hline $\mathrm{AC}$ & $\langle--\rangle$ & PAV & $-0,621$ & 0,032 & 50,45 & 0,000 \\
\hline $\mathrm{AC}$ & $\langle-->$ & TR & 0,519 & 0,035 & 13,73 & 0,000 \\
\hline $\mathrm{AC}$ & $\langle--\rangle$ & $\mathrm{PC}$ & $-0,669$ & 0,030 & 54,77 & 0,000 \\
\hline $\mathrm{AC}$ & $\langle--\rangle$ & IN & 0,534 & 0,035 & 13,44 & 0,000 \\
\hline PAV & <--> & TR & $-0,542$ & 0,034 & 44,76 & 0,000 \\
\hline PAV & <--> & $\mathrm{PC}$ & 0,721 & 0,028 & 9,82 & 0,000 \\
\hline PAV & <--> & IN & $-0,611$ & 0,032 & 49,64 & 0,000 \\
\hline TR & $<-->$ & $\mathrm{PC}$ & $-0,614$ & 0,032 & 49,88 & 0,000 \\
\hline TR & <--> & IN & 0,496 & 0,036 & 14,16 & 0,000 \\
\hline PC & <--> & IN & $-0,613$ & 0,032 & 49,80 & 0,000 \\
\hline
\end{tabular}

Để phân tích thống kê các mô hình đo lường và cấu trúc, nghiên cứu này đã sử dụng phần mềm Smart PLS cho kỹ thuật mô hình cấu trúc tuyến tính (SEM) [71]. Mô hình lý thuyết của nghiên cứu đã đo lường đến mối liên kết giữa các biến thuộc về lợi ích cảm nhận và chi phí cảm nhận với biến tiềm ẩn ở dạng cấu tạo là giá trị cảm nhận và mô hình cấu trúc nắm bắt các mối quan hệ nhân quả được đưa ra với giả thuyết giữa các cấu trúc nghiên cứu [72]. Không giống như AMOS và LISREL là các phương pháp dựa trên hiệp phương sai, Smart PLS là một kỹ thuật dựa trên hồi quy bắt nguồn từ phân tích đường dẫn. Smart PLS đã nổi lên như một cách tiếp cận mạnh mẽ để nghiên cứu các mô hình nguyên nhân liên quan đến nhiều cấu trúc với nhiều chỉ số [73]. Smart PLS - một phương pháp dựa trên thành phần, có khả năng mô hình hóa các cấu trúc tiềm ẩn không bị ảnh hưởng bởi lỗi đo lường trong các điều kiện không quy tắc. Về mặt này, phương pháp lấy mẫu Bootstrapping đã được sử dụng để kiểm tra ý nghĩa thống kê của các mối quan hệ. Quy trình này đòi hỏi phải tạo ra 3000 các trường hợp mẫu phụ được chọn ngẫu nhiên, với sự thay thế, từ dữ liệu gốc. Kết quả mô hình SEM được trình bày ở Bảng 7. Theo Hu và Bentler [74] nếu mô hình nhận được giá trị SRMR nhỏ hơn 0,1 thì được xem là phù hợp với dữ liệu thực tế. Ngoài ra, Hair và cônng $s u ̛$ [75] cũng cho rằng chỉ số SRMR là chỉ số goodness of fit của mô hình PLS-SEM có thể được sử dụng để tránh hiện tượng sai lệch thông số trong mô hình. Như vậy, với giá trị SRMR =0,097 < 0,1, mô hình nghiên cứu được kết luận phù hợp với dữ liệu thị trường. 
Nhằm có thể suy rộng kết quả nghiên cứu ra tổng thể, mô hình cần được tiến hành kiểm định lại độ tin cậy. Đề tài sử dụng kỹ thuật bootstrapping với cỡ mẫu lặp lại là 3000 quan sát $(\mathrm{n}=3000)$ với cỡ mẫu ban đầu là 597 quan sát. Kết quả ước lượng từ 3000 quan sát ở bảng 7 cho thấy trọng số gốc có ý nghĩa với trọng số trung bình của bootstrapping vì tất cả trọng số đều nằm trong khoảng tin cậy $95 \%$. Như vậy, các ước lượng trong mô hình có thể kết luận là đáng tin cậy.

Bảng 7. Kết quả Bootstrapping và kiểm định giả thuyết

\begin{tabular}{|l|r|r|r|r|r|r|r|l|}
\hline & $\begin{array}{c}\text { Trọng số } \\
\text { gốc }\end{array}$ & $\begin{array}{c}\text { Trọng số } \\
\text { bootstrapping }\end{array}$ & $\begin{array}{c}\text { Độ lệch } \\
\text { chuẩn }\end{array}$ & $\begin{array}{l}\text { Kiểm } \\
\text { định } \mathrm{t}\end{array}$ & $2,50 \%$ & $97,50 \%$ & $\begin{array}{c}\text { Giả } \\
\text { thuyết }\end{array}$ & Kết luận \\
\hline TR -> PV & 0,237 & 0,236 & 0,01 & 22,675 & 0,216 & 0,257 & H1a & Chấp nhận \\
\hline IN -> PV & 0,326 & 0,327 & 0,022 & 14,847 & 0,289 & 0,375 & H1b & Chấp nhận \\
\hline AC -> PV & 0,234 & 0,233 & 0,011 & 20,922 & 0,212 & 0,255 & H1c & Chấp nhận \\
\hline PC -> PV & $-0,302$ & $-0,303$ & 0,017 & 17,678 & $-0,337$ & $-0,27$ & H2a & Chấp nhận \\
\hline PAV -> PV & $-0,256$ & $-0,255$ & 0,014 & 17,703 & $-0,283$ & $-0,226$ & H2b & Chấp nhận \\
\hline
\end{tabular}

Như đã trình bày, nghiên cứu có năm giả thuyết cần được kiểm định. Kết quả ước lượng mối quan hệ giữa các khái niệm nghiên cứu cho thấy cả năm nhân tố nghiên cứu đều tạo ra giá trị cảm nhận cho khách hàng khi cung cấp thông tin với độ tin cậy $99 \%(\mathrm{p}<0,001)$. Bảng 7 cho thấy giả thuyết H1a về sự tin tưởng ảnh hưởng đồng biến với giá trị cảm nhận được chấp nhận $(\beta=0,237 ; \mathrm{t}>2,56)$. H1b về mối quan hệ đồng biến giữa sự quan tâm về thông tin và giá trị cảm nhận cũng được chấp nhận $(\beta=0,326 ; \mathrm{t}>2,56)$. Kết quả thống kê cũng ủng hộ giả thuyết $\mathrm{H} 1 \mathrm{c}$, đó là khả năng kiểm soát về thông tin có ảnh hưởng tích cực đến giá trị cảm nhận của khách hàng khi sử dụng các ứng dụng di động để mua hàng hóa $(\beta=0,234 ; \mathrm{t}>2,56)$. Kết quả nghiên cứu cũng khẳng định quan tâm về sự riêng tư $(\beta=-0,302 ; \mathrm{t}>2,56)$ và nhận thức về lỗ hổng ( $\beta$ $=-0,256 ; \mathrm{t}>2,56$ ) sẽ tác động tiêu cực đến giá trị cảm nhận của khách hàng khi họ cung cấp thông tin để mua hàng thông qua các thiết bị di động bằng ứng dụng. Điều này ủng hộ giả thuyết $\mathrm{H} 2 \mathrm{a}$ và $\mathrm{H} 2 \mathrm{~b}$.

\section{THẢO LUẬN VÀ HÀM Ý KẾT QUẢ}

Mục tiêu của nghiên cứu này nhằm xác định giá trị cảm nhận của khách hàng khi cung cấp thông tin trong thương mại di động với sự đánh đổi giữa những lợi ích và chi phí về bảo mật thông tin riêng tư. Kết quả nghiên cứu định tính và khảo sát định lượng với kích thước mẫu là 597 quan sát để kiểm định mô hình cấu trúc tuyến tính, trong đó, giá trị cảm nhận như một biến cấu tạo bậc hai bị ảnh hưởng bởi các yếu tố lợi ích cảm nhận và chi phí cảm nhận khi cung cấp thông tin trên các ứng dụng điện thoại để mua hàng và giao dịch trực tuyến. Kết quả đã cho chúng ta một số hàm ý về mặt lý thuyết và thực tiễn như sau:

Một là, ngoài các phần thưởng bằng tiền để kích thích người tiêu dùng cung cấp thông tin [51] thì các yếu tố lợi ích về tinh thần như sự quan tâm thông tin, sự tin tưởng, và khả năng kiểm soát của người dùng cũng là một động lực khiến khách hàng cảm thấy giá trị của việc cung cấp thông tin khi mua hàng trên các thiết bị di động. Nghiên cứu này cũng khẳng định lại tính phù hợp của kết quả lý thuyết phương tiện và giá trị sau cùng [13]. Trong đó, sự quan tâm của khách hàng về thông tin tạo ra giá trị cảm nhận cao nhất $(\beta=0,326)$, tiếp theo là sự tin tưởng của khách hàng đối với dịch vụ trên các thiết bị di động $(\beta=0,237)$, cuối cùng là khả năng kiểm soát $(\beta=0,234)$. Yếu tố sự quan tâm thông tin là yếu tố khách hàng có thể kiểm soát được và là nhu cầu thông tin của khách hàng, trong khi đó, yếu tố về sự tin tưởng và khả năng kiểm soát chỉ phản ánh sự phụ thuộc của khách hàng vào nhận thức cá nhân với những yếu tố như danh tiếng, sự hiểu biết cá nhân và thông qua các chính sách an ninh của doanh nghiệp; điều này phù hợp với lý thuyết nhận thức xã hội trong môi trường kinh doanh và công nghệ hiện nay [76]. Để tạo ra sự nhận thức giá trị cao khi cung cấp thông tin, các doanh nghiệp cần xây dựng danh tiếng trong thị trường thương mại di động bằng những chính sách rõ ràng và thực hiện đúng cam kết với khách hàng về việc bảo mật thông tin, cũng 
như xử lý các trường hợp rủi ro về thông tin một cách công khai và minh bạch. Ngoài ra, doanh nghiệp cũng nên tăng cường về giá trị trên hệ thống ứng dụng như cung cấp các thông tin về khuyến mãi, thông tin về giảm giá qua ứng dụng, trang mạng xã hội của khách hàng. Tạo ra khả năng cá nhân hóa đối với từng khách hàng trong việc quảng cáo, cũng như đề xuất các sản phẩm. Hơn nữa, các ứng dụng thương mại di động cần được thiết kế phải có sự đồng ý của khách hàng mới được sử dụng, truy vấn thông tin (có các popup thông báo trên màn hình các thiết bị di động).

Hơn nữa, nghiên cứu cũng đã chỉ ra được mối quan hệ nghịch biến giữa quan tâm sự riêng tư cũng như nhận thức về lỗ hổng đối với giá trị cảm nhận. Mức độ quan tâm riêng tư $(\beta=-0,302)$ có ảnh hưởng nghịch biến đến giá trị cảm nhận cao hơn so với nhận thức về lỗ hổng $(\beta=-0,256)$. Kết quả nghiên cứu phản ánh đúng bản chất tâm lý của người tiêu dùng là e ngại khi liên quan đến sự riêng tư [46], cũng như những rủi ro về thông tin khi giao dịch [77]. Do đó, doanh nghiệp cần phải có những chính sách về an ninh thông tin để tạo ra giá trị cao hơn trong nhận thức của khách hàng. Sử dụng giao thức an toàn khi giao dịch trên các phương tiên di động như SSL hoặc SET nhằm đảm bảo về dữ liệu trên đường truyền cũng như tại máy chủ nhà cung cấp dịch vụ. Ngoài ra, chỉ thu thập những thông tin cần thiết cho việc giao hàng cũng nhu chăm sóc khách hàng, những thông tin thu thập phải được sự đồng ý của khách hàng. Bên cạnh đó, cần phải đăng ký với Bộ Công Thương các chứng nhận về bán hàng trực tuyến thông qua ứng dụng di động, cũng như, chứng nhận giao dịch điện tử an toàn. Các doanh nghiệp có thể liên kết với các bên thứ ba đáng tin cậy như TRUSTee để có được chứng chỉ đảm bảo an toàn khi giao dịch. Ngoài ra, chính sách sử dụng thông tin của khách hàng cũng cần được công khai trên hệ thống của doanh nghiệp.

\section{HAN CHẾ VÀ HƯỚNG NGHIÊN CỨU TIẾP THEO}

Tuy đã cố gắng thực hiện một cách khoa học và hoàn thiện nhất, nhưng nghiên cứu vẫn còn một số hạn chế. Thứ nhất, nghiên cứu chỉ mới thực nghiệm chung chung thương mại di động, mặc dù thương mại di động là một lĩnh vực tương đối rộng với nhiều loại mặt hàng và hình thức kinh doanh. Thứ hai, về phương pháp chọn mẫu, vì lý do thời gian và khung dữ liệu, nên tác giả chỉ có thể chọn mẫu phi xác suất có đánh giá, điều này làm giảm độ tin cậy của nghiên cứu. Thứ ba, nghiên cứu chỉ tập trung vào giá trị, lợi ích và chi phí thông tin trong giao dịch thương mại di động.

Những nghiên cứu tiếp theo có thể khắc phục một số hạn chế của nghiên cứu này bằng cách tập trung vào một doanh nghiệp cụ thể hay hàng hóa cụ thể trong nghiên cứu. Bên cạnh đó, có thể xây dựng khung chọn mẫu và chọn mẫu để điều tra theo phương pháp xác suất để tạo độ tin cậy cao hơn cho nghiên cứu. Một số biến nghiên cứu có thể đườc tăng cường để có thể tạo ra nghiên cứu mở rộng, đặc biệt là các biến nghiên cứu về lợi ích cảm nhận, chi phí cảm nhận ở các khía cạnh khác ngoài khía cạnh thông tin trong giao dịch thương mại điện tử.

\section{TÀI LIÊU THAM KHẢO}

[1] M. S. Chhonker, D. Verma và A. K. Kar, Review of technology adoption frameworks in mobile commerce, Procedia computer science, vol. 122, pp. 888-895, 2017.

[2] S. Hillman và C. Neustaedter, Trust and mobile commerce in North America, Computers in Human Behavior, vol. 70, pp. 10-21, 2017.

[3] W. T. Rupp và A. D. Smith, Mobile commerce: New revenue machine or black hole?, Business Horizons, vol. 45, pp. 26-26, 2002.

[4] Nielsen, Điện thoại thông minh tiếp tục trên đà phát triển ở cả khu vực thành thị và nông thôn, 2017.

[5] Nielsen. Nguời Việt sủ dụng hơn ba ngày làm việc để online mỗi tuần. Internet: https://www.nielsen.com/vn/vi/insights/2016/vietnam-cross-platform-2016.html, ngày truy cập: 24/06/2018 
[6] Cục Thương mại điện tử và kinh tế số, Sách trắng Thương mại điện tử Việt Nam 2018, www.idea.gov.vn, 2018.

[7] VECITA, Báo cáo Thương mại điện tử Việt Nam 2017, Cục Thương mại điện tử và Công nghệ thông tin, Bộ Công Thương, 2017.

[8] J. R. Turner và M. Gellman, Encyclopedia of Behavioral Medicine: Springer, 2013.

[9] S. Forsythe, C. Liu, D. Shannon và L. C. Gardner, Development of a scale to measure the perceived benefits and risks of online shopping, Journal of interactive marketing, vol. 20, pp. 55-75, 2006.

[10] H. Ko, J. Jung, J. Kim và S. W. Shim, Cross-cultural differences in perceived risk of online shopping, Journal of Interactive Advertising, vol. 4, pp. 20-29, 2004.

[11] P. Kotler và K. L. Keller, Marketing management vol. 15e: Pearson, 2016.

[12] R. N. Bolton và K. N. Lemon, A dynamic model of customers' usage of services: Usage as an antecedent and consequence of satisfaction, Journal of marketing research, pp. 171-186, 1999.

[13] V. A. Zeithaml, Consumer perceptions of price, quality, and value: a means-end model and synthesis of evidence, The Journal of marketing, pp. 2-22, 1988.

[14] H. Ahola, H. Oinas-Kukkonen và T. Koivumaki, "Customer delivered value in a web-based supermarket," in System Sciences, 2000. Proceedings of the 33rd Annual Hawaii International Conference on, 2000, p. 10 pp. vol. 2.

[15] E. Turban, J. Outland, D. King, J. K. Lee, T.-P. Liang và D. C. Turban, Electronic Commerce 2018: A Managerial and Social Networks Perspective: Springer, 2017.

[16] R. Blaise, M. Halloran và M. Muchnick, Mobile commerce competitive advantage: A quantitative study of variables that predict m-commerce purchase intentions, Journal of Internet Commerce, vol. 17, pp. 96-114, 2018.

[17] A. K. Ghosh và T. M. Swaminatha, Software security and privacy risks in mobile e-commerce, Communications of the ACM, vol. 44, pp. 51-57, 2001.

[18] O. E. Oxford, Oxford English Dictionary: Oxford: Oxford University Press, 2009.

[19] W. Van Eerde và H. Thierry, Vroom's expectancy models and work-related criteria: A meta-analysis, Journal of applied psychology, vol. 81, p. 575, 1996.

[20] N. V. T. Trường, "A Study on the Typology of Internet Users Based on Privacy Concerns and Their Different Styles of Privacy Calculus Process," Doctor, Department of Business Administration, Soongsil University, Korea, 2012.

[21] T. Dinev và P. Hart, "Privacy concerns and Internet use--a model of trade-off factors " in Academy of Management Proceedings, 2003, pp. D1-D6.

[22] T. Tsu Wei, G. Marthandan, A. Yee-Loong Chong, K.-B. Ooi và S. Arumugam, What drives Malaysian mcommerce adoption? An empirical analysis, Industrial Management \& Data Systems, vol. 109, pp. 370-388, 2009.

[23] J. J. Cronin Jr, M. K. Brady và G. T. M. Hult, Assessing the effects of quality, value, and customer satisfaction on consumer behavioral intentions in service environments, Journal of retailing, vol. 76, pp. 193-218, 2000.

[24] J. Sweeney và G. Soutar, Consumer perceived value: The development of a multiple item scale, Journal of retailing, vol. 77, pp. 203-220, 2001. 

TRỰC TUYẾN: TRƯỜNG HỢP THƯƠNG MẠI DI ĐỘNG TẠI VIỆT NAM

[25] A. Parasuraman, L. L. Berry và V. A. Zeithaml, Understanding customer expectations of service, MIT Sloan Management Review, vol. 32, p. 39, 1991.

[26] C.-H. Lin, P. J. Sher và H.-Y. Shih, Past progress and future directions in conceptualizing customer perceived value, International Journal of Service Industry Management, vol. 16, pp. 318-336, 2005.

J. Carlos Fandos Roig, J. Sanchez Garcia, M. Angel Moliner Tena và J. Llorens Monzonis, Customer perceived value in banking services, International Journal of Bank Marketing, vol. 24, pp. 266-283, 2006.

[28] L. Ang, C. Dubelaar và B.-C. Lee, To trust or not to trust? A model of internet trust from the customer's point of view, BLED 2001 Proceedings, p. 43, 2001.

[29] R. J. Bies, Privacy and procedural justice in organizations, Social Justice Research, vol. 6, pp. 69-86, 1993.

[30] T. Dinev và P. Hart, An extended privacy calculus model for e-commerce transactions, Information systems research, vol. 17, pp. 61-80, 2006.

[31] H. Van der Heijden, T. Verhagen và M. Creemers, Understanding online purchase intentions: contributions from technology and trust perspectives, European journal of information systems, vol. 12, pp. 41-48, 2003.

[32] S. Pace, A grounded theory of the flow experiences of Web users, International journal of human-computer studies, vol. 60, pp. 327-363, 2004.

[33] S. L. Jarvenpaa và P. A. Todd, Is there a future for retailing on the Internet, Electronic marketing and the consumer, vol. 1, pp. 139-154, 1997.

[34] T. P. Novak, D. L. Hoffman và Y.-F. Yung, Measuring the customer experience in online environments: A structural modeling approach, Marketing science, vol. 19, pp. 22-42, 2000.

[35] T. L. Childers, C. L. Carr, J. Peck và S. Carson, Hedonic and utilitarian motivations for online retail shopping behavior, Journal of retailing, vol. 77, pp. 511-535, 2001.

[36] E. Goffman, Stigma: Notes on the management of spoiled identity: Simon and Schuster, 2009.

[37] V. J. Derlega và A. L. Chaikin, Privacy and self-disclosure in social relationships, Journal of Social Issues, vol. 33, pp. 102-115, 1977.

[38] E. F. Stone và D. L. Stone, Privacy in organizations: Theoretical issues, research findings, and protection mechanisms, Research in personnel and human resources management, vol. 8, pp. 349-411, 1990.

[39] G. J. Nowak và J. Phelps, Direct marketing and the use of individual-level consumer information: Determining how and when "privacy" matters, Journal of Interactive Marketing, vol. 11, pp. 94-108, 1997.

[40] P. Wang và L. A. Petrison, Direct marketing activities and personal privacy. A consumer survey, Journal of Direct Marketing, vol. 7, pp. 7-19, 1993.

[41] J. Phelps, G. Nowak và E. Ferrell, Privacy concerns and consumer willingness to provide personal information, Journal of Public Policy \& Marketing, vol. 19, pp. 27-41, 2000.

[42] A. Gurung, X. Luo và M. Raja, An empirical investigation on customer's privacy perceptions, trust and security awareness in E-commerce environment, Journal of Information Privacy and Security, vol. 4, pp. 4260, 2008.

[43] R. N. Stone và K. Grønhaug, Perceived risk: Further considerations for the marketing discipline, European Journal of marketing, vol. 27, pp. 39-50, 1993.

[44] N. K. Malhotra, S. S. Kim và J. Agarwal, Internet users' information privacy concerns (IUIPC): The construct, the scale, and a causal model, Information systems research, vol. 15, pp. 336-355, 2004. 
[45] H. Xu, X. R. Luo, J. M. Carroll và M. B. Rosson, The personalization privacy paradox: An exploratory study of decision making process for location-aware marketing, Decision support systems, vol. 51, pp. 42-52, 2011.

[46] B. T. Khoa, Nghiên cứu nhận thức bảo vệ thông tin riêng tư của người mua hàng trực tuyến tại TP.HCM, Tạp chí Khoa học và Công nghệ, vol. 2, pp. 66-76, 2017.

[47] C. D. Raab, The distribution of privacy risks: Who needs protection?, The information society, vol. 14, pp. 263-274, 1998.

[48] H. J. Smith, S. J. Milberg và S. J. Burke, Information privacy: measuring individuals' concerns about organizational practices, MIS quarterly, pp. 167-196, 1996.

[49] M. E. Budnitz, Privacy protection for consumer transactions in electronic commerce: why self-regulation is inadequate, SCL Rev., vol. 49, p. 847, 1997.

[50] M. J. Culnan và S. Milberg, The second exchange: Managing customer information in marketing relationships, 1998.

[51] I.-H. Hann, K.-L. Hui, T. Lee và I. Png, Online information privacy: Measuring the cost-benefit trade-off, ICIS 2002 proceedings, p. 1, 2002.

[52] J. Gutman, Means-end chains as goal hierarchies, Psychology \& marketing, vol. 14, pp. 545-560, 1997.

[53] C. M. Chiu, E. T. Wang, Y. H. Fang và H. Y. Huang, Understanding customers' repeat purchase intentions in B2C e-commerce: the roles of utilitarian value, hedonic value and perceived risk, Information Systems Journal, vol. 24, pp. 85-114, 2014.

[54] J. Gutman, A means-end chain model based on consumer categorization processes, The Journal of Marketing, pp. 60-72, 1982.

[55] D. Cox và S. Rich, Perceived risk and consumer decision-making: The case of telephone shopping, Journal of marketing research, pp. 32-39, 1964.

[56] L. F. Cunningham, J. Gerlach và M. D. Harper, Assessing perceived risk of consumers in Internet airline reservations services, Journal of Air Transportation, vol. 9, 2004.

[57] F. Rabiee, Focus-group interview and data analysis, Proceedings of the nutrition society, vol. 63, pp. 655660, 2004.

[58] D. Silverman, Qualitative research: Sage, 2016.

[59] W. L. Neuman, Social research methods: Qualitative and quantitative approaches: Pearson education, 2013.

[60] J. F. Hair, R. E. Anderson, B. J. Babin và W. C. Black, Multivariate data analysis: A global perspective vol. 7: Pearson Upper Saddle River, NJ, 2010.

[61] T. Kowatsch và W. Maass, "Critical privacy factors of internet of things services: An empirical investigation with domain experts," in Knowledge and Technologies in Innovative Information Systems: Springer, 2012, pp. 200-211.

[62] K. D. Martin, A. Borah và R. W. Palmatier, Data privacy: Effects on customer and firm performance, Journal of Marketing, vol. 81, pp. 36-58, 2017.

[63] H. Xu, T. Dinev, H. J. Smith và P. Hart, Examining the formation of individual's privacy concerns: Toward an integrative view, ICIS 2008 proceedings, p. 6, 2008.

[64] R. B. Kline, Principles and practice of structural equation modeling: Guilford publications, 2015.

[65] J. C. Nunnally và I. Bernstein, The assessment of reliability, Psychometric theory, vol. 3, pp. 248-292, 1994. 

TRỰC TUYẾN: TRƯỜNG HỢP THƯƠNG MẠI DI ĐỘNG TẠI VIỆT NAM

[66] J. C. Anderson và D. W. Gerbing, Structural equation modeling in practice: A review and recommended twostep approach, Psychological bulletin, vol. 103, p. 411, 1988.

[67] C. Fornell và D. F. Larcker, Evaluating structural equation models with unobservable variables and measurement error, Journal of marketing research, pp. 39-50, 1981.

[68] P. M. Bentler và D. G. Bonett, Significance tests and goodness of fit in the analysis of covariance structures, Psychological bulletin, vol. 88, p. 588, 1980.

[69] H. W. Marsh, J. R. Balla và K.-T. Hau, An evaluation of incremental fit indices: A clarification of mathematical and empirical properties, Advanced structural equation modeling: Issues and techniques, pp. 315-353, 1996.

[70] J.-B. E. Steenkamp và H. C. Van Trijp, The use of LISREL in validating marketing constructs, International Journal of Research in marketing, vol. 8, pp. 283-299, 1991.

[71] C. Ringle, S. Wende và A. Will, SmartPLS version 2.0 M2, Hamburg, Germany: Institute for Operations Management and Organization at the University of Hamburg, 2005.

[72] W. W. Chin và P. R. Newsted, Structural equation modeling analysis with small samples using partial least squares, Statistical strategies for small sample research, vol. 1, pp. 307-341, 1999.

[73] R. Chinomona và J. Surujlal, The influence of student internship work experience on their self-improvement and professionalism in Sport Management: sport management, African Journal for Physical Health Education, Recreation and Dance, vol. 18, pp. 885-899, 2012.

[74] L. t. Hu và P. M. Bentler, Cutoff criteria for fit indexes in covariance structure analysis: Conventional criteria versus new alternatives, Structural equation modeling: a multidisciplinary journal, vol. 6, pp. 1-55, 1999.

[75] J. F. Hair, J. Henseler, T. K. Dijkstra và M. Sarstedt, Common beliefs and reality about partial least squares: comments on Rönkkö and Evermann, 2014.

[76] C.-M. Chiu, M.-H. Hsu và E. T. Wang, Understanding knowledge sharing in virtual communities: An integration of social capital and social cognitive theories, Decision support systems, vol. 42, pp. 1872-1888, 2006.

[77] M. S. Featherman và P. A. Pavlou, Predicting e-services adoption: a perceived risk facets perspective, International journal of human-computer studies, vol. 59, pp. 451-474, 2003.

Ngày nhận bài: 02/02/2019

Ngày chấp nhận đăng: 20/02/2019 